\title{
Influencia de las medidas preventivas ergonómicas en el desarrollo de secuelas por patología no traumática del hombro
}

\author{
Influence of Ergonomic Preventive Resources in \\ Development of Sequels Due to Non-Traumatic \\ Diseases of the Shoulder
}

\author{
Gloria M. Rodríguez-Blanes ${ }^{1}$ \\ José Rafael Lobato-Cañón ${ }^{2,3}$ \\ José Sánchez-Payá ${ }^{4,5}$ \\ José Ramón Ausó-Pérez ${ }^{6}$ \\ Antonio Francisco J. Cardona-Llorens ${ }^{2}$ \\ ${ }^{1}$ Centro de Salud Pública de Alcoy, Alicante. Consellería de Sanidad Universal y Salud Pública, Genera- \\ litat Valenciana, España. \\ ${ }^{2}$ Departamento de Patología y Cirugía. Universidad Miguel Hernández, San Juan de Alicante, Alicante, \\ España. \\ ${ }^{3}$ Instituto Nacional de la Seguridad Social, Alicante, España. \\ ${ }^{4}$ Servicio de Medicina Preventiva. Hospital General Universitario de Alicante, Alicante, España. \\ ${ }^{5}$ Instituto de Investigación Sanitaria y Biomédica de Alicante (ISABIAL), Alicante, España. \\ ${ }^{6}$ Servicio de Cirugía Ortopédica y Traumatología, Hospital de Villajoyosa, Alicante, España.
}

Fechas · Dates

Recibido: 2019.10.21

Aceptado: 2020.01.23

Publicado: 2020.04.15
Correspondencia $\cdot$ Corresponding Author

G.M. Rodríguez-Blanes

Centro de Salud Pública de Alcoy. Avda. Alameda de Camilo Sesto, nº41, 03803

Alcoy, Alicante, España.

g.rodriguez@umh.es. 


\section{Resumen}

Objetivo: Describir las secuelas referidas en los casos atendidos por patología no traumática del hombro . Y conocer cómo se asocian con la presencia de secuelas las características sociodemográficas, disponer de conocimientos en prevención de riesgos laborales y los requerimientos ergonómicos del puesto de trabajo.

Métodos: Estudio observacional transversal en una población de 345 pacientes atendidos durante un año en las consultas de un centro de atención primaria de salud en Alicante. Mediante la prueba de la Ji-cuadrado se estudiaron las asociaciones entre las secuelas y el resto de variables.

Resultados: El 53,9\% ( $n=186)$ de los participantes refirieron presentar secuelas derivadas, sobre todo dolor ocasional asociado o no a limitación de la movilidad. Son factores de riesgo: un bajo nivel de estudios, actividades de limpieza y sector industrial, alzar los brazos por encima del hombro y la monotonía en las tareas. Sin embargo, son factores protectores: el sexo masculino, disponer de información sobre los riesgos laborales, equipos de protección individual, existencia de Servicio de Prevención de Riesgos Laborales, evaluación de riesgos, plan de prevención y los reconocimientos médicos periódicos. Conocer los riesgos del puesto se mostró como factor de protección independiente frente al desarrollo de secuelas (ORa=0,2; IC95\%=0,1-0,4). Sin embargo, alzar el brazo por encima del hombro, se comporta como factor de riesgo independiente para el desarrollo de secuelas por patología del hombro (ORa=2,1; IC95\%=1,0-4,3).

Conclusiones: La combinación de estrategias ergonómicas y formativas parecen útiles para la prevención del desarrollo de secuelas a nivel del hombro.

Palabras clave: Secuelas, trastornos musculoesqueléticos, hombro, prevención, riesgos laborales, Atención Primaria

\section{Abstract}

Objective: To describe the sequelae referred to the cases attended by non-traumatic diseases of the shoulder. To know how sociodemographic characteristics, preventive knowledge about the workplace and ergonomic requirements are associated with the presence of sequelae.

Methods: Cross-sectional observational study in a population of 345 patients attended for a year in the consultations of a primary health care center in Alicante. Through the Chi-square test, the associations between the sequels and the rest of the variables were studied.

Results: $53.9 \%$ ( $n=186)$ of the participants reported having sequelae derived, especially occasional pain associated or not with limitation of joint mobility. They are risk factors: a low level of studies, cleaning activities and industrial sector, raising the arm over the shoulder and the monotony in the workplace. However, they are protective factors: male sex, having information on occupational risks, Personal Protective Equipment, the existence of a Labor Risk Prevention Service, Risk Assessment, Prevention Plan and periodic medical examinations. Knowing the risks of the position was shown as an independent protection factor against the development of secondary sequelae in the shoulder (ORa=0,2; IC95\%=0,1-0,4). However, to raise the arm above the shoulder, behaving as an independent risk factor for the development of sequelae due to shoulder pathology (ORa=2,1; IC95\%=1,0-4,3). 
Conclusions: The combination of ergonomic and training strategies seems useful in the prevention of the development of shoulder-level sequelae.

Keywords: Sequels, musculoskeletal disorders, shoulder, prevention, occupational health, Primary Health Care

\section{Introducción}

El dolor de hombro es un motivo frecuente de consulta por patología musculoesquelética tanto en atención primaria( ${ }^{(1)}$, como a nivel laboral(2). Su prevalencia aproximada es del 7-26\% en población general(3,4), sugiriéndose fracciones atribuibles poblacionales por exposiciones laborales del $24 \%^{(2)}$. Su principal causa es la tendinitis o la tendinosis del manguito rotador, responsable de más de dos tercios de los casos de hombro doloroso(5). La Organización Mundial de la Salud (OMS) considera los trastornos del hombro "enfermedades relacionadas con el trabajo"(6), y tanto en Europa como particularmente en España, se incluye dentro del cuadro de enfermedades profesionales, entre otras, la patología tendinosa crónica del manguito de los rotadores ${ }^{(7)}$.

Debido a la gran utilización del hombro en las actividades de la vida diaria y a nivel laboral, esta articulación es susceptible de sufrir gran variedad de patologías tanto debidas a traumatismos directos como indirectos, o las derivadas de posturas forzadas o movimientos repetitivos. Éstas podrían ocasionar una laxitud global adquirida de la cápsula y causar una inestabilidad multidireccional, consistente en la presencia de dolor durante la translación glenohumeral( ${ }^{(8)}$. Por lo tanto, las lesiones del hombro a menudo provocan una discapacidad funcional considerable.

Una secuela puede definirse como: "estadio último que en su evolución logra alcanzar una lesión y que a partir de este momento ya no es previsible en ciencia que se experimenten más cambios o transformaciones en el estado anatómico funcional que se observa, siendo ya inútil cualquier terapéutica pretendiendo mejoría”(9) ; es decir, la aparición de una secuela conlleva algo más que una pérdida de movilidad, es la alteración de una función determinada en un individuo concreto con su edad, sexo, profesión, circunstancias y repercusión psicológica(10).

En el desarrollo de las secuelas pueden intervenir múltiples factores de tipo físico y/o psicosocial. Son factores de riesgo ergonómicos conocidos para el desarrollo de patología a nivel del hombro: la manipulación manual de cargas, tareas que supongan elevar los brazos por encima del nivel de los hombros, cargar sobre un único hombro, movimientos repetitivos, posturas forzadas y vibraciones ${ }^{(2,3)}$. La exposición simultánea a varios de estos factores parece estar relacionada con la persistencia del dolor, y la sobrecarga física percibida parece retrasar la reincorporación laboral(2). En cuanto al manejo de la patología del hombro ocupacional, existe creciente evidencia que apoya un abordaje bio-psico-social, incluyendo un retorno temprano al trabajo para obtener los mejores resultados ${ }^{(2,3,11)}$. También las intervenciones educativas centradas en empoderar y aumentar la autoeficacia del trabajador a través de la información (incluyendo los factores de riesgo laboral 
a los que están expuestos), pueden obtener beneficios a través de cambios de

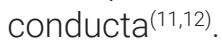

Los trastornos musculoesqueléticos ocasionan importante costes socio-económicos para el trabajador, para la empresa y para la sociedad, tanto directos como indirectos, estimándose que representan a nivel de miembro superior entre el 0,5 y el 2\% del Producto Interior Bruto del país. Dentro de los costes directos se incluyen los seguros, indemnizaciones, gastos médicos y de gestión. Los indirectos son derivados de las bajas laborales e incluyen la contratación y formación de nuevos empleados, disminución de la productividad y efectos sobre la producción y calidad del trabajo(13).

Los objetivos de este estudio fueron: 1) Describir las secuelas referidas en los casos atendidos por patología no traumática del hombro . 2) Conocer la asociación entre las variables sociodemográficas, disponer de conocimientos en prevención de riesgos laborales del puesto y los requerimientos ergonómicos del puesto de trabajo, con la presencia de secuelas por patología osteomuscular no traumática del hombro.

\section{Métodos}

Diseño y Población de Estudio: se realizó un estudio observacional de tipo transversal llevado a cabo en el período comprendido entre febrero de 2008 y mayo de 2009. La población de estudio fue constituida por los/las pacientes trabajadores atendida a lo largo de un año en un Centro de Atención Primaria perteneciente al Departamento de Salud de Alicante-Hospital General.

Criterios de inclusión y exclusión: fueron incluidos todos aquellos pacientes que consultaron con su médico de familia por dolor en la región del hombro a lo largo del año. Para su selección se incluyeron los diagnósticos correspondientes con los códigos CIE-9-MC 726.1, 726.10, 726.11, 726.12, 726.13, 726.19, 726.2, 719.01, $719.41,719.61,719.81,719.91,840.3-840.6$ que correspondían a las siguientes patologías: enfermedades por fatiga e inflamación de las vainas tendinosas, de tejidos peritendinosos e inserciones musculares y tendinosas en el hombro (tendinitis, rotura de tendones, síndrome subacromial), enfermedades de las bolsas serosas debida a la presión (bursitis del hombro) y dolor articular del hombro respectivamente.

Se excluyeron todos aquellos pacientes que presentaron: edad menor de 16 años o mayor de 65; diagnósticos secundarios a traumatismos directos o de origen artrósico; no haber desarrollado ninguna actividad laboral remunerada durante el año previo o más; o manifestar su desacuerdo a participar en el estudio.

Variables: se recopilaron datos sobre las siguientes variables:

Demográficas (variables independientes): sexo, edad y nivel de estudios.

Puesto de trabajo: actual, clasificado por Ocupación y Actividad Económica, horas trabajadas diarias y semanales referidas. 
Conocimientos sobre prevención de riesgos laborales (variables independientes): información acerca de los riesgos del puesto desempeñado, uso de Equipos de Protección Individual (EPI), existencia de Servicio de Prevención de Riesgos Laborales (SPRL) asociado a la empresa, evaluación de riesgos, existencia de un plan de Prevención, y realización de vigilancia de la salud periódica.

Requerimientos ergonómicos del puesto (variables independientes): adquisición de posturas forzadas, utilización y elevación de brazos, empleo de herramientas vibratorias, manejo de cargas, necesidad de golpear superficies y emplear la fuerza, movimientos repetidos, consideración del trabajo como monótono y la existencia y duración de las pausas.

Secuelas referidas: existencia o no de secuelas (variable dependiente), y tipo de secuelas.

Procedimiento de recogida de datos: Todos los participantes respondieron a una encuesta semiestructurada autodiseñada para la recogida de datos, tras ser informados del motivo del estudio y dar su consentimiento informado verbal a participar. De los 514 pacientes iniciales, se excluyeron aquellos que no cumplían el criterio de tener actividad laboral $(n=54)$; aquellos con quienes no fue posible contactar ( $n=109$ ); a los que manifestaron su deseo a no participar en el estudio $(n=5)$; y 1 caso que se excluyó por defunción debida otra causa no relacionada.

Análisis estadístico: la descripción de las variables se llevó a cabo mediante la frecuencia absoluta y relativa en porcentajes de cada una de las categorías de las variables. Los sujetos estudiados (345 casos) se dividieron en dos grupos únicamente en función de la existencia o no de secuelas referidas por el trabajador. Para el estudio de asociaciones entre las secuelas y el resto de variables se empleó la prueba de la Ji-cuadrado. La magnitud de la asociación se calculó mediante la Odds Ratio de prevalencia (OR) con un intervalo de confianza (IC) al 95\%. Para estudiar el efecto independiente de las variables explicativas se realizó un análisis multivariante de tipo regresión logística. El nivel de significación utilizado en los contrastes de hipótesis fue de $p<0,05$. El cálculo de los test estadísticos se realizó con el programa SPSS v. 15.

Consideraciones éticas: Todos los participantes fueron informados del motivo del estudio y les fue solicitado su consentimiento para participar en el mismo. No se admitió en ningún caso el consentimiento por representación. Asimismo, se les informó de la posibilidad de revocar su consentimiento en cualquier momento. Para garantizar la confidencialidad y el anonimato de los participantes se eliminaron los datos identificativos personales, asignando a cada caso un número consecutivo de orden. Los autores declaran la no existencia de conflictos de intereses.

\section{Resultados}

El $53,9 \%$ de los participantes en el estudio $(n=186)$ refirieron presentar secuelas debido a la patología a nivel del hombro. Destaca el dolor ocasional $(n=82,44,1 \%)$, seguido por una limitación de la movilidad asociada a dolor $(n=39,21,0 \%)$, o la 
existencia de dolor crónico ( $n=26,14,0 \%)$. El tratamiento más frecuentemente utilizado ( $n=188,54,5 \%$ de los casos) fue el combinado (farmacológico+rehabilitación). Sin embargo, sólo el 2,9\% de los casos ( $n=10)$ referían presentar una Incapacidad Permanente derivada reconocida, siendo la más frecuente la Incapacidad Permanente Total para su profesión habitual $(n=7,70,0 \%)$ (Tabla 1).

Tabla 1: Características básicas de los pacientes que presentan patología del hombro y frecuencia de secuelas.

\begin{tabular}{|c|c|}
\hline & Total $(n=345)$ \\
\hline & $\mathrm{N}(\%)$ \\
\hline \multicolumn{2}{|l|}{ Tipo de Tratamiento } \\
\hline Médico & $138(40,0 \%)$ \\
\hline Quirúrgico & $12(3,5 \%)$ \\
\hline Combinado (méd+RHB) & $188(54,5 \%)$ \\
\hline Ninguno & $7(2,0 \%)$ \\
\hline \multicolumn{2}{|l|}{ Tratamiento específico } \\
\hline Sutura manguito & $12(3,5 \%)$ \\
\hline Electroterapia + Gimnasia & $170(49,3 \%)$ \\
\hline Infiltraciones & $40(11,6 \%)$ \\
\hline Otros & $123(35,7 \%)$ \\
\hline \multicolumn{2}{|l|}{ Secuelas } \\
\hline Sí & $186(53,9 \%)$ \\
\hline \multicolumn{2}{|l|}{ Tipos de secuelas $(n=186)$} \\
\hline Parestesias + limitación movilidad & $1(0,5 \%)$ \\
\hline Dolor + parestesias & $2(1,1 \%)$ \\
\hline Dolor crónico & $26(14,0 \%)$ \\
\hline Ánimo deprimido & $1(0,5 \%)$ \\
\hline Disminución fuerza + limitación movilidad + dolor & $3(1,6 \%)$ \\
\hline Disminución fuerza + limitación movilidad & $3(1,6 \%)$ \\
\hline Limitación movilidad + dolor & $39(21,0 \%)$ \\
\hline Disminución fuerza & $2(1,1 \%)$ \\
\hline Dolor ocasional & $82(44,1 \%)$ \\
\hline Limitación en grandes esfuerzos & $11(5,9 \%)$ \\
\hline Limitación en las AVD & $16(8,6 \%)$ \\
\hline No & $159(46,1 \%)$ \\
\hline \multicolumn{2}{|l|}{ Incapacidad Permanente } \\
\hline Sí & $10(2,9 \%)$ \\
\hline \multicolumn{2}{|l|}{ Tipos de Incapacidad $(n=10)$} \\
\hline Parcial & $2(20,0 \%)$ \\
\hline Permanente total & $7(70,0 \%)$ \\
\hline Permanente absoluta & $1(10,0 \%)$ \\
\hline No & 335 (97,1\%) \\
\hline
\end{tabular}


Se asociaron a una mayor probabilidad de presentar una secuela por patología del hombro: el bajo nivel de estudios, las actividades relacionadas con la limpieza y la industria, así como las ocupaciones de limpiadoras, artesanos, agricultores y ganaderos, y los mecánicos, soldadores y montadores industriales. También se mostraron como factores de riesgo los requerimientos ergonómicos en el puesto de alzar los brazos por encima del hombro y la monotonía en las tareas desempeñadas. Sin embargo, se mostraron como factores protectores frente a desarrollar secuelas por patología a nivel del hombro: el sexo masculino, disponer de información sobre los riesgos laborales, Equipos de Protección Individual (EPIs), existencia de un Servicio de Prevención de Riesgos Laborales asociado a la empresa, tener Evaluación de Riesgos, Plan de prevención y la realización de reconocimientos médicos periódicos (Tablas 2, 3 y 4).

Tras el análisis Multivariante, estar informado frente a los riesgos del puesto de trabajo se mostró como un factor de protección independiente frente al desarrollo de secuelas derivadas en el hombro (ORa=0,2; IC95\%=0,1-0,4). Sin embargo, que un requerimiento habitual sea alzar el brazo por encima del hombro, se comporta como factor de riesgo independiente para el desarrollo de secuelas por patología no traumática del hombro $(\mathrm{ORa}=2,1 ; \mathrm{IC} 95 \%=1,0-4,3)$ (Tablas 3 y 4$)$.

Tabla 2: Análisis de la asociación entre variables sociodemográficas y secuelas en los casos estudiados

\begin{tabular}{|c|c|c|c|c|c|c|}
\hline & $\begin{array}{c}\text { Con Secuelas } \\
(n=186)\end{array}$ & $\begin{array}{c}\text { Sin Secuelas } \\
\quad(n=159)\end{array}$ & $\begin{array}{c}\text { OR } \\
\text { (IC 95\%) }\end{array}$ & $\mathrm{p}$ & ORa (IC 95\%) & $P$ \\
\hline \multicolumn{7}{|l|}{ Sexo } \\
\hline Hombre & $24,2 \%(45)$ & $41,5 \%(66)$ & $0,4(0,3-0,7)$ & 0,001 & $1,1(0,5-2,4)$ & N.S. \\
\hline Mujer & $75,8 \%(141)$ & $58,5 \%(93)$ & 1 & & 1 & \\
\hline \multicolumn{7}{|l|}{ Edad } \\
\hline $51-65$ & $71,0 \%(132)$ & $63,5 \%(101)$ & $1,4(0,9-2,2)$ & 0,141 & & \\
\hline $16-50$ & $29,0 \%(54)$ & $36,5 \%(58)$ & 1 & & & \\
\hline \multicolumn{7}{|c|}{ Nivel de estudios } \\
\hline $\begin{array}{l}\text { Sin estudios/ } \\
\text { Primarios }\end{array}$ & $65,1 \%(121)$ & $41,5 \%(66)$ & $2,6(1,7-4,1)$ & $<0,001$ & $1,2(0,6-2,1)$ & N.S. \\
\hline $\begin{array}{l}\text { Secundarios/ } \\
\text { Formación } \\
\text { Profesional/ } \\
\text { Superiores }\end{array}$ & $34,9 \%(65)$ & $58,5 \%(93)$ & 1 & & 1 & \\
\hline \multicolumn{7}{|c|}{ Actividad Económica } \\
\hline $\begin{array}{l}\text { Construcción, } \\
\text { Agricultura, } \\
\text { Ganadería y } \\
\text { Pesca }\end{array}$ & $4,8 \%(9)$ & $9,4 \%(15)$ & $1,1(0,4-3,2)$ & N.S. & $0,7(0,1-7,5)$ & N.S. \\
\hline $\begin{array}{l}\text { Comercio y } \\
\text { Hostelería }\end{array}$ & $12,4 \%(23)$ & $11,9 \%(19)$ & $2,3(0,9-5,4)$ & 0,067 & $0,5(0,1-3,2)$ & N.S. \\
\hline
\end{tabular}




\begin{tabular}{|c|c|c|c|c|c|c|}
\hline & $\begin{array}{c}\text { Con Secuelas } \\
(n=186)\end{array}$ & $\begin{array}{c}\text { Sin Secuelas } \\
(n=159)\end{array}$ & $\begin{array}{c}\text { OR } \\
\text { (IC 95\%) }\end{array}$ & $p$ & ORa (IC 95\%) & $P$ \\
\hline $\begin{array}{l}\text { Sanidad y } \\
\text { Servicios sociales }\end{array}$ & $4,8 \%(9)$ & $12,6 \%(20)$ & $0,8(0,3-2,3)$ & N.S. & - & - \\
\hline $\begin{array}{l}\text { Transporte y } \\
\text { almacén }\end{array}$ & $8,1 \%(15)$ & $8,8 \%(14)$ & $2,0(0,8-5,2)$ & 0,158 & $2,3(0,5-9,9)$ & N.S \\
\hline Limpieza & $46,8 \%(87)$ & $22,0 \%(35)$ & $4,6(2,2-9,7)$ & $<0,001$ & $0,3(0,03-2,5)$ & N.S \\
\hline Servicios & $5,9 \%(11)$ & $11,9 \%(19)$ & $1,1(0,4-2,9)$ & N.S. & $0,6(0,1-3,1)$ & N.S \\
\hline Industria & $9,1 \%(17)$ & $5,7 \%(9)$ & $3,5(1,3-9,8)$ & 0,016 & $1,2(0,2-7,7)$ & N.S \\
\hline $\begin{array}{l}\text { Administración, } \\
\text { banca y seguros. } \\
\text { Enseñanza }\end{array}$ & $8,1 \%(15)$ & $17,6 \%(28)$ & 1 & & 1 & \\
\hline \multicolumn{7}{|l|}{ Ocupación } \\
\hline Albañiles & $3,2 \%(6)$ & $8,2 \%(13)$ & $3,7(0,4-36,4)$ & N.S. & - & \\
\hline Conductores & $3,8 \%(7)$ & $6,3 \%(10)$ & $5,6(0,6-55-2)$ & 0,141 & & \\
\hline Sanitarios & $3,8 \%(7)$ & $11,3 \%(18)$ & $3,1(0,3-29,5)$ & N.S. & $0,3(0,02-4,2)$ & N.S \\
\hline Profesores & $1,1 \%(2)$ & $5,0 \%(8)$ & $2,0(0,1-26,6)$ & N.S. & & \\
\hline Limpiadoras & $51,6 \%(96)$ & $23,9 \%(38)$ & $20,2(2,4-166,3)$ & 0,005 & & - \\
\hline Comerciales & $9,1 \%(17)$ & $11,3 \%(18)$ & $7,5(0,8-66,6)$ & 0,069 & & - \\
\hline $\begin{array}{l}\text { Administrativos y } \\
\text { directivos }\end{array}$ & $10,2 \%(19)$ & $16,4 \%(26)$ & $5,8(0,7-50,5)$ & 0,110 & & \\
\hline $\begin{array}{l}\text { Artesanos, } \\
\text { agricultores y } \\
\text { ganaderos }\end{array}$ & $8,6 \%(16)$ & $4,4 \%(7)$ & $18,2(1,9-174,5)$ & 0,012 & & - \\
\hline $\begin{array}{l}\text { Defensa y } \\
\text { seguridad }\end{array}$ & $1,1 \%(2)$ & $1,9 \%(3)$ & $5,3(0,3-82,5)$ & N.S. & & - \\
\hline $\begin{array}{l}\text { Mecánicos, } \\
\text { soldadores, } \\
\text { montadores } \\
\text { industriales }\end{array}$ & $7,0 \%(13)$ & $6,3 \%(10)$ & $10,4(1,1-96,9)$ & 0,040 & & \\
\hline $\begin{array}{l}\text { Informáticos e } \\
\text { ingenieros }\end{array}$ & $0,5 \%(1)$ & $5,0 \%(8)$ & 1 & & 1 & \\
\hline
\end{tabular}

Horas trabajo diarias

\begin{tabular}{lllcll}
\hline$\geq 9 h$ & $9,1 \%(17)$ & $6,9 \%(11)$ & $1,2(0,5-2,8)$ & N.S. & - \\
$8 \mathrm{~h}$ & $46,8 \%(87)$ & $52,2 \%(83)$ & $0,8(0,5-1,3)$ & N.S. & - \\
$\leq 7 \mathrm{~h}$ & $44,1 \%(82)$ & $40,9 \%(65)$ & 1 & \\
\hline
\end{tabular}

Horas trabajo semanales

\begin{tabular}{lllclll}
\hline$>40 \mathrm{~h}$ & $10,8 \%(20)$ & $7,5 \%(12)$ & $1,3(0,6-2,8)$ & N.S. & - & - \\
$40 \mathrm{~h}$ & $45,7 \%(85)$ & $53,5 \%(85)$ & $0,8(0,5-1,2)$ & N.S. & - \\
$<40 \mathrm{~h}$ & $43,5 \%(81)$ & $39,0 \%(62)$ & 1 & & \\
\hline
\end{tabular}

N.S.: $p>0.200$. Ajustado por: Sexo, Nivel de estudios, Actividad económica, Información de los riesgos, Equipos de protección, Servicio de prevención, Evaluación de riesgos, Plan de prevención, Vigilancia de salud, Alzar el hombro, Herramientas vibratorias, Trabajo monótono. 
Tabla 3: Análisis de la asociación entre conocimientos sobre prevención de los riesgos del puesto de trabajo y presencia de secuelas en los casos estudiados

\begin{tabular}{lllllll}
\hline & $\begin{array}{l}\text { Con } \\
\text { Secuelas } \\
(\mathbf{n}=\mathbf{1 8 6})\end{array}$ & $\begin{array}{l}\text { Sin } \\
\text { Secuelas } \\
(\mathbf{n}=\mathbf{1 5 9})\end{array}$ & OR (IC 95\%) & P & ORa (IC 95\%) & P \\
\hline Información riesgos & & & & & & \\
\hline Si & $28,5 \%(53)$ & $72,3 \%(115)$ & $0,1(0,1-0,2)$ & $<0,001$ & $0,2(0,1-0,4)$ & $<0,001$ \\
No & $71,5 \%(133)$ & $27,7 \%(44)$ & 1 & & 1 & \\
\hline
\end{tabular}

\section{Equipos de protección}

\begin{tabular}{|c|c|c|c|c|c|c|}
\hline Si & $34,9 \%(65)$ & $52,8 \%(84)$ & $0,5(0,3-0,7)$ & 0,001 & $1,3(0,6-2,7)$ & N.S. \\
\hline No & $65,1 \%(121)$ & $47,2 \%(75)$ & 1 & & 1 & \\
\hline
\end{tabular}

\section{Servicio de Prevención}

\begin{tabular}{lllllll}
\hline $\mathrm{Si}$ & $32,3 \%(60)$ & $68,6 \%(109)$ & $0,3(0,1-0,3)$ & $<0,001$ & $1,7(0,5-5,1)$ & N.S. \\
$\mathrm{No}$ & $67,7 \%(126)$ & $31,4 \%(50)$ & 1 & 1 & \\
\hline
\end{tabular}

\begin{tabular}{|c|c|c|c|c|c|c|}
\hline \multicolumn{7}{|c|}{ Evaluación de riesgos } \\
\hline Si & $23,7 \%(44)$ & $57,2 \%(91)$ & $0,2(0,1-0,4)$ & $<0,001$ & $0,9(0,2-3,5)$ & N.S. \\
\hline No & $76,3 \%$ (142) & $42,8 \%(68)$ & 1 & & 1 & \\
\hline
\end{tabular}

\begin{tabular}{lllllll}
\hline Plan de prevención & & & & & \\
\hline Si & $23,7 \%(44)$ & $56,6 \%(90)$ & $0,2(0,1-0,4)$ & $<0,001$ & $0,7(0,2-2,9)$ & N.S. \\
No & $76,3 \%(142)$ & $43,4 \%(69)$ & 1 & & 1 & \\
\hline
\end{tabular}

\begin{tabular}{lllllll}
\hline Vigilancia de salud & & & & & \\
\hline Si & $38,2 \%(71)$ & $71,1 \%(113)$ & $0,2(0,2-0,4)$ & $<0,001$ & $0,7(0,3-1,6)$ & N.S. \\
No & $61,8 \%(115)$ & $28,9 \%(46)$ & 1 & & 1 & \\
\hline
\end{tabular}

N.S.: $p>0.200$. Ajustado por: Sexo, Nivel de estudios, Actividad económica, Información de los riesgos, Equipos de protección, Servicio de prevención, Evaluación de riesgos, Plan de prevención, Vigilancia de salud, Alzar el hombro, Herramientas vibratorias, Trabajo monótono.

Tabla 4: Análisis de la asociación entre requerimientos ergonómicos del puesto de trabajo y presencia de secuelas

\begin{tabular}{|c|c|c|c|c|c|c|}
\hline & $\begin{array}{l}\text { Con Secuelas } \\
\quad(n=186)\end{array}$ & $\begin{array}{c}\text { Sin Secuelas } \\
\quad(n=159)\end{array}$ & OR (IC 95\%) & $P$ & ORa (IC 95\%) & $P$ \\
\hline \multicolumn{7}{|c|}{ Posturas forzadas } \\
\hline $\mathrm{Si}$ & $94,6 \%(176)$ & $95,0 \%(151)$ & $0,9(0,4-2,4)$ & N.S. & & \\
\hline No & $5,4 \%(10)$ & $5,0 \%(8)$ & 1 & & & \\
\hline \multicolumn{7}{|c|}{ Brazo utilizado } \\
\hline Único & $12,4 \%(23)$ & $17,6 \%(28)$ & $0,7(0,4-1,2)$ & N.S. & & \\
\hline Ambos & $87,6 \%(163)$ & $82,4 \%(131)$ & 1 & & & \\
\hline \multicolumn{7}{|c|}{ Brazo dominante } \\
\hline Derecho & $96,2 \%(179)$ & $96,9 \%(154)$ & $0,8(0,3-2,7)$ & N.S. & - & \\
\hline Izquierdo & $3,8 \%(7)$ & $3,1 \%(5)$ & 1 & & & \\
\hline \multicolumn{7}{|c|}{ Alzar hombro } \\
\hline $\mathrm{Si}$ & $81,7 \%(152)$ & $67,3 \%(107)$ & $2,2(1,3-3,6)$ & 0,002 & $2,1(1,0-4,3)$ & 0,042 \\
\hline
\end{tabular}




\begin{tabular}{|c|c|c|c|c|c|c|}
\hline & $\begin{array}{c}\text { Con Secuelas } \\
(n=186)\end{array}$ & $\begin{array}{c}\text { Sin Secuelas } \\
(n=159)\end{array}$ & OR (IC 95\%) & $P$ & ORa (IC 95\%) & $P$ \\
\hline No & $18,3 \%(34)$ & $32,7 \%(52)$ & 1 & & 1 & \\
\hline \multicolumn{7}{|c|}{ Herramientas vibratorias } \\
\hline $\mathrm{Si}$ & $14,0 \%(26)$ & $21,4 \%(34)$ & $0,6(0,3-1,0)$ & 0,070 & $0,5(0,2-1,3)$ & N.S \\
\hline No & $86,0 \%(160)$ & $78,6 \%(125)$ & 1 & & 1 & \\
\hline \multicolumn{7}{|c|}{ Golpear superficies } \\
\hline $\mathrm{Si}$ & $10,2 \%(19)$ & $15,7 \%(25)$ & $0,6(0,3-1,1)$ & N.S. & & \\
\hline No & $89,8 \%(167)$ & $84,3 \%(134)$ & 1 & & & \\
\hline \multicolumn{7}{|c|}{ Postura de trabajo } \\
\hline Sentado & $14,0 \%(26)$ & $21,4 \%(34)$ & $0,6(0,3-1,1)$ & 0,092 & & \\
\hline De pie & $39,8 \%(74)$ & $36,5 \%(58)$ & $1,0(0,6-1,6)$ & N.S. & & \\
\hline $\begin{array}{l}\text { Alternados y } \\
\text { cuclillas }\end{array}$ & $46,2 \%(86)$ & $42,1 \%(67)$ & 1 & & & \\
\hline \multicolumn{7}{|c|}{ Girar cuello-columna } \\
\hline $\mathrm{Si}$ & $92,5 \%(172)$ & $91,8 \%(146)$ & $1,1(0,5-2,4)$ & N.S. & & \\
\hline No & $7,5 \%(14)$ & $8,2 \%(13)$ & 1 & & & \\
\hline \multicolumn{7}{|c|}{ Cargas $>15 \mathrm{Kg}$. } \\
\hline $\mathrm{Si}$ & $29,6 \%(55)$ & $31,4 \%(50)$ & $0,9(0,6-1,4)$ & N.S. & & \\
\hline No & $70,4 \%(131)$ & $68,6 \%(109)$ & 1 & & & \\
\hline \multicolumn{7}{|c|}{ Uso fuerza física } \\
\hline $\mathrm{Si}$ & $15,1 \%(28)$ & $18,2 \%(29)$ & $0,8(0,4-1,4)$ & N.S. & & \\
\hline No & $84,9 \%(158)$ & $81,8 \%(130)$ & 1 & & & \\
\hline \multicolumn{7}{|c|}{ Movimientos repetitivos } \\
\hline Si & $92,5 \%(172)$ & $89,9 \%(143)$ & $1,4(0,6-2,9)$ & N.S. & & \\
\hline No & $7,5 \%(14)$ & $10,1 \%(16)$ & 1 & & & \\
\hline \multicolumn{7}{|c|}{ Trabajo monótono } \\
\hline Si & $50,0 \%(93)$ & $37,7 \%(60)$ & $1,6(1,1-2,5)$ & 0,022 & $1,0(0,6-1,7)$ & N.S \\
\hline No & $50,0 \%(93)$ & $62,3 \%(99)$ & 1 & & 1 & \\
\hline \multicolumn{7}{|l|}{ Pausas } \\
\hline $\mathrm{Si}$ & $86,0 \%(160)$ & $89,9 \%(143)$ & $0,7(0,3-1,3)$ & N.S. & & \\
\hline No & $14,0 \%(26)$ & $10,1 \%(16)$ & 1 & & & \\
\hline \multicolumn{7}{|c|}{ Duración de las pausas } \\
\hline$<30$ minutos & $50,0 \%(80)$ & $57,3 \%(82)$ & $0,7(0,5-1,2)$ & N.S. & & \\
\hline$\geq 30$ minutos & $50,0 \%(80)$ & $42,7 \%(61)$ & 1 & & & \\
\hline \multicolumn{7}{|c|}{ Posibles ausencias } \\
\hline $\mathrm{Si}$ & $65,6 \%(122)$ & $71,7 \%(114)$ & $0,7(0,5-1,2)$ & N.S. & - & - \\
\hline No & $34,4 \%(64)$ & $28,3 \%(45)$ & 1 & & & \\
\hline
\end{tabular}

N.S.: $p>0.200$. Ajustado por: Sexo, Nivel de estudios, Actividad económica, Información de los riesgos, Equipos de protección, Servicio de prevención, Evaluación de riesgos, Plan de prevención, Vigilancia de salud, Alzar el hombro, Herramientas vibratorias, Trabajo monótono. 


\section{Discusión}

La valoración funcional de las alteraciones del hombro se ha orientado históricamente hacia la existencia de dolor, alteraciones en la movilidad, rigidez o inestabilidad articular. En trabajos más recientes se valora la percepción del paciente sobre la repercusión en su estado general de salud ${ }^{(14)}$. Los datos obtenidos en este estudio se basan únicamente en la percepción referida por los entrevistados, siendo las principales secuelas referidas: dolor ocasional, dolor crónico, disminución de la fuerza o de la movilidad, ánimo deprimido, o combinaciones entre ellas.

El dolor en la región del hombro es altamente recurrente ${ }^{(4)}$. No obstante en muchas ocasiones, se vive como una experiencia de corta duración por parte del paciente, y probablemente esté infraestimada, por lo que no es fácil conocer la existencia real de secuelas( ${ }^{(2)}$. Además, se observan grandes diferencias en las tasas de recuperación entre estudios ${ }^{(15)}$. Aquellos basados en consultas médicas (normalmente atención primaria) informan de menores proporciones de curación que los estudios basados en el ámbito laboral(15). Un trabajo calcula que el dolor de hombro de origen laboral tiene una duración media aproximada de 10 meses, aunque un $25 \%$ de los pacientes refieren persistencia del mismo a los 22 meses de iniciarse el proceso(16).

Según nuestros resultados, el bajo nivel de estudios se muestra como un factor de riesgo para presentar secuelas por patología a nivel del hombro, y el sexo masculino actuaría como factor de protección. Según la literatura, la edad avanzada y un nivel educativo bajo actúan como factores de riesgo para los trastornos musculoesqueléticos que causen incapacidad permanente ${ }^{(17,18)}$. La edad avanzada se asociaría con una recuperación más lenta, actuando como un importante predictor para una pensión por incapacidad(2,17), aunque nuestros resultados no muestran significación estadística. El papel de la educación parece consistente y puede explicarse por una manera inadecuada de afrontar la enfermedad ${ }^{(18)}$.

En nuestro estudio se comportaron como factores de riesgo frente al desarrollo de secuelas en el hombro las siguientes ocupaciones: limpiadoras; artesanos, agricultores y ganaderos; y, mecánicos, soldadores y montadores industriales, pertenecientes a las actividades económicas de limpieza y el sector industrial. No se ha encontrado evidencias en la literatura que analicen específicamente las secuelas musculoesqueléticas en el hombro en relación a la ocupación y/o actividad económica, aunque un estatus educativo y socioeconómico más bajo se asocia a peores condiciones en el puesto de trabajo, con una mayor demanda física, y, por lo tanto, a mayores tasas de discapacidad por daño musculoesquelético(17).

De acuerdo a nuestros resultados, actuarían como factores de riesgo para el desarrollo de secuelas los siguientes requerimientos del puesto de trabajo: alzar el brazo por encima del nivel del hombro, y la monotonía en las tareas del puesto. Además, alzar el brazo por encima del hombro sería un factor independiente para el desarrollo de secuelas, concordando con las evidencias encontradas en la literatura ${ }^{(3,19)}$. Según otros estudios, la exposición simultánea a, al menos, dos de los siguientes factores: trabajos manuales, alzar los brazos por encima del nivel 
del hombro y el uso de herramientas vibratorias manuales; se mostraron como factores de riesgo para la permanencia del dolor en el hombro, mientras que un trabajo sedentario mejora la perspectiva de estar libre de síntomas, probablemente debido a la falta de exposición a otras sobrecargas mecánicas ${ }^{(15,20)}$. Un alto nivel de dolor también actúa como factor pronóstico negativo ${ }^{(21)}$. El trabajo repetitivo se ha mostrado como otro factor pronóstico negativo, al igual que la percepción del trabajo como monótono ${ }^{(22,23)}$. En esta línea, se ha encontrado alguna evidencia de asociación entre la sobrecarga en el trabajo y la persistencia del dolor en el hombro, que apunta que la percepción de una sobrecarga física puede dificultar la vuelta al trabajo, enfatizando la necesidad de una adaptación del trabajo en los programas para la reincorporación al puesto(24,25).

En relación a los conocimientos del trabajador (información y formación) para prevenir los riesgos laborales y su influencia en el desarrollo de secuelas, todas las variables analizadas se comportan como factores de protección. Además, el haber sido informado de los riesgos del puesto de trabajo se muestra como un factor independiente de protección por sí solo. Estos datos concuerdan con los obtenidos por otros autores, que observaron cómo una reducción de los factores de riesgo biomecánicos mediante cambios organizativos y ajustes en el puesto de trabajo, podrían potencialmente reducir los trastornos de cuello y hombro(26). También la educación podría potencialmente actuar reduciendo los factores de riesgo mediante el cambio en el comportamiento de los empleados ${ }^{(27)}$. Por todo lo anteriormente expuesto, como recomendación preventiva aplicable en cualquier puesto de trabajo, debemos insistir en el papel de la información y formación para prevenir los riesgos laborales específicos, ya es el único factor de protección independiente modificable en todos los casos. Otra recomendación preventiva en cuanto a la presencia de secuelas a nivel del hombro sería actuar sobre las posturas forzadas, más concretamente, reduciendo en la medida de lo posible el requerimiento ergonómico de alzar los brazos por encima del nivel del hombro (el otro factor de riesgo independiente), aunque con la salvedad de tratarse de un factor no susceptible de abordaje en todos los casos a través de cambios organizativos y ajustes en el puesto de trabajo.

Las limitaciones de este estudio son las propias de un diseño transversal. La información sobre la exposición se recogió retrospectivamente, por lo que podría ocasionar un sesgo de memoria en los participantes y ambigüedad temporal. Además, la valoración de las secuelas se basa en la percepción referida por el paciente, que podría haber influido en las respuestas obtenidas, ocasionando un posible sesgo de deseabilidad social, tal vez contrarrestado por el anonimato de los participantes. La tasa media de respuestas de los participantes fue del $67,1 \%$, por lo que no nos permite asegurar la representatividad de la muestra y exige prudencia al extrapolar los resultados. No se recogieron antecedentes de lesiones deportivas o aficiones con utilización de los hombros, ni realización de tareas domésticas que podrían originar la patología sin asociación con el trabajo. Otra potencial limitación propia podría ser la existencia de un potencial sesgo de selección derivado de la no inclusión de casos atendidos en el centro de salud pero con codificación diagnóstica errónea. 
Más de la mitad de los casos refirieron presentar secuelas derivadas de la patología, principalmente dolor asociado o no a limitación de la movilidad articular. La exposición combinada a varios requerimientos ergonómicos o alzar el brazo por encima del hombro de manera habitual son factores de riesgo para desarrollar secuelas. Sin embargo, la combinación de estrategias preventivas y conocer los riesgos del puesto son factores de protección, por lo que la combinación de estrategias ergonómicas y formativas podrían ser útiles en la prevención del desarrollo de secuelas a nivel de la articulación del hombro.

\section{Agradecimientos}

A todas las personas que accedieron a participar en el estudio.

\section{Bibliografia}

1. Marin-Gomez M, Navarro-Collado MJ, Peiro S, Trenor-Gomis C, Paya-Rubio A, Bernal-Delgado E, et al. La calidad de la atención al hombro doloroso. Audit clínico. Gac Sanit. 2006;20:116-23.

2. Shanahan EM, Sladek R. Shoulder pain at the workplace. Best Pract Res Clin Rheumatol. 2011;25:59-68.

3. Linaker $\mathrm{CH}$, Walker-Bone K. Shoulder disorders and occupation. Best Pract Res Clin Rheumatol. 2015;29:405-23.

4. Luime J, Koes B, Hendriksen I, Burdorf A, Verhagen A, Miedema H, et al. Prevalence and incidence of shoulder pain in the general population; a systematic review. Scand J Rheumatol. 2004;33:73-81.

5. Murphy RJ, Carr AJ. Shoulder pain. BMJ Clin Evid. 2010;2010:pii:1107.

6. World Health Organization. Identification and control of work-related diseases: report of a WHO expert committee [meeting held in Geneva from 28 November to 2 December 1983]. Geneva: World Health Organization; 1985.

7. Real Decreto 1299/2006, de 10 de noviembre, por el que se aprueba el cuadro de Enfermedades Profesionales en el sistema de la Seguridad Social y se establecen criterios para su notificación y registro. Real Decreto 1299/2006 (19 Dic 2006).

8. Miralles-Marrero RC, Segura-Mata J. Traumatismos de la cintura escapular. En: Miralles-Marrero RC, coordinador. Valoración del daño corporal en el aparato locomotor. Barcelona: Masson; 2001. p. 75-101.

9. Hinojal-Fonseca R. Daño corporal: fundamentos y métodos de valoración médica. Oviedo: Arcano Medicina; 1996.

10. Miralles-Marrero RC. Introducción. En: Miralles-Marrero RC , coordinador. Valoración del dano corporal en el aparato locomotor. Barcelona: Masson; 2001. p. 3-6. 
11. Larsson B, Søgaard K, Rosendal L. Work related neck-shoulder pain: a review on magnitude, risk factors, biochemical characteristics, clinical picture and preventive interventions. Best Pract Res Clin Rheumatol. 2007;21:447-63.

12. Hutting N, Engels JA, Staal JB, Heerkens YF, Nijhuis-van der Sanden MW. Development of a self-management intervention for employees with complaints of the arm, neck and/or shoulder (CANS): a focus group study with experts. J Occup Med Toxicol. 2015;10:9.

13. Schneider E, Irastorza X, Copsey S. OSH [Occupational safety and health] in figures: workrelated musculoskeletal disorders in the EU - facts and figures. Luxembourg: Publications Office of the European Union; 2010.

14. Liang MH, Fossel AH, Larson MG. Comparisons of five health status instruments for orthopedic evaluation. Med Care. 1990;28:632-42.

15. Grooten W. Work and neck/shoulder pain: risk and prognostic factors. Stockholm: Karolinska Institutet. 2006.

16. Bonde JP, Mikkelsen S, Andersen J, Fallentin N, Bælum J, Svendsen SW, et al. Prognosis of shoulder tendonitis in repetitive work: a follow up study in a cohort of Danish industrial and service workers. Occup Environ Med. 2003;60:e8.

17. Gjesdal S, Bratberg E, Mæland JG. Musculoskeletal impairments in the Norwegian working population: the prognostic role of diagnoses and socioeconomic status: a prospective study of sickness absence and transition to disability pension. Spine (Phila Pa 1976). 2009;34:1519-25.

18. van Eijsden-Besseling MD, van den Bergh $K A$, Staal JB, de Bie RA, van den Heuvel WJ. The course of nonspecific work-related upper limb disorders and the influence of demographic factors, psychologic factors, and physical fitness on clinical status and disability. Arch Phys Med Rehabil. 2010;91:862-7.

19. Harkness E, Macfarlane G, Nahit E, Silman A, McBeth J. Mechanical and psychosocial factors predict new onset shoulder pain: a prospective cohort study of newly employed workers. Occup Environ Med. 2003;60:850-7.

20. Grooten WJA, Mulder M, Josephson M, Alfredsson L, Wiktorin C. The influence of work-related exposures on the prognosis of neck/shoulder pain. Eur Spine J. 2007;16:2083-91.

21. Viikari-Juntura E, Martikainen $R$, Luukkonen $R$, Mutanen $P$, Takala E, Riihimäki H. Longitudinal study on work related and individual risk factors affecting radiating neck pain. Occup Environ Med. 2001;58:345-52.

22. Descatha A, Roquelaure $Y$, Chastang J-F, Evanoff B, Cyr D, Leclerc A. Description of outcomes of upper-extremity musculoskeletal disorders in workers highly exposed to repetitive work. J Hand Surg Am. 2009;34:890-5.

23. Herin F, Vézina M, Thaon I, Soulat J-M, Paris C. Predictors of chronic shoulder pain after 5years in a working population. Pain. 2012;153:2253-9. 
24. Lötters F, Burdorf A. Prognostic factors for duration of sickness absence due to musculoskeletal disorders. Clin J Pain. 2006;22:212-21.

25. Miranda H, Viikari-Juntura E, Martikainen R, Takala E, Riihimäki H. A prospective study of work related factors and physical exercise as predictors of shoulder pain. Occup Environ Med. 2001;58:528-34.

26. Buckle PW, Devereux JJ. The nature of work-related neck and upper limb musculoskeletal disorders. Appl Ergon. 2002;33:207-17.

27. Leonard DM. The effectiveness of intervention strategies used to educate clients about prevention of upper extremity cumulative trauma disorders. Work. 2000;14:151-7. 